\title{
Research and Technological Innovation in CALL ${ }^{1}$
}

\author{
Mike Levy \\ School of Languages and Linguistics, Griffith University, Brisbane, \\ Queensland, Australia
}

Almost continuous innovation in the area of new technologies and applications in second language teaching and learning can make research especially challenging. In longer-term projects, for example, the researcher may find that by the end of the project the technology that has been central to it has been surpassed or outmoded. While some precautionary steps can be taken, such as focusing on underlying principles, the technology in use still remains critical. This is why the drive for deeper principles and theoretical insights in CALL research becomes so important. However, it is also a matter of the kind of research being attempted, for example whether it is preliminary and exploratory, aiming to evaluate a very new technology or application, or whether it is more refined and focussed, incorporating the use of a technology or application that is relatively stable and well established. This paper focuses on the impact of technological innovation in CALL research, while accepting that pedagogical innovation and curriculum innovation are also contributing factors. The paper describes exemplary projects and strategies for research planning where the aim is to sustain longer-term research agendas alongside shorter-term research goals which, while still reflecting broader principles, are still responsive to rapid innovation and development.

doi: $10.2167 /$ illt069.0

Keywords: CALL, Computer Assisted Language Learning, technological innovation, research

\section{Introduction}

There is a potential tension at the heart of all research that involves new technology and language learning. This tension arises because good CALL research takes time and requires the researcher to carefully build on prior work in order better to understand the phenomena in focus; often this necessitates a long-term research agenda. Unfortunately, the technology itself is constantly evolving and changing; it does not wait for the researcher, and, as a rule, it evolves rapidly and independently, in its own commercial timeframe.

The commercial imperative is a powerful one. When associated with the competitiveness between educational institutions, the motivation for technological innovation and change is further amplified. Whatever the technological innovations and uptake in the wider world, we might expect the institution to provide a critical gate-keeping role, but, in the main, aside from teachers asking students to switch off their mobile phones, technological innovation in the institution has tended to follow patterns of technology adoption in the wider world. In the Western world at least, when new hardware or software 
applications become available they are quickly adopted in the institution: old machines are replaced, older software applications are upgraded or replaced. The teaching staff or faculty of the institution are rarely consulted.

When a more cautious approach is suggested, observers often say of new technologies, 'It will happen anyway', reflecting the position of the technological determinist. In other words, regardless of our efforts to modify or regulate the innovation, or act on what research findings may tell us, the new technology will be adopted. It appears that technological innovation is not contingent upon research results, positive or negative. Technological innovation in society at large and in education appears to arise in a parallel world that does not take heed of the results of good research studies. We saw this in the 1980s when numerous studies were conducted to identify the benefits and limitations of the word processor compared to pen and paper for second language writing (e.g. see Pennington, 1991). In spite of some important reservations that were clearly outlined in findings from a range of research studies concerning, chiefly, planning strategies, reading differences on screen and on paper, and problems for non-native speakers when using the spellchecker or the grammar checker, the application was widely adopted anyway, and after time it seemed that these issues of concern were forgotten. Users simply adapt; at least that appears to be the widespread assumption or belief. ${ }^{2}$

In the meantime, technological innovation has continued rapidly and in ways that have been surprisingly sustained. Perhaps one might have expected some relative stability after the 1990s with the advent of the Internet, the World Wide Web, multimedia and a variety of communication technologies that, after decades of experimentation, finally converged and provided the infrastructure to enable users to communicate with one another using integrated forms of text, image and video while at a distance. But the rapid developments continue, with MP3s, podcasting, wikis and blogs being some of the recent innovations, and the new mobile technologies that integrate an increasingly large number of features within the same device. On the other hand, some new technologies proved not to be successful and disappeared without trace. In a memorable piece on the next decade in CALL written in the mid 1980s, which correctly predicted many technological developments, Phillips (1985) talked about the impact and potential value of holograms in CALL, but we have heard little about them since. Numerous brands of computer and software applications appeared and disappeared in educational institutions in the 1980s and early 1990s until, in recent years, we have moved, for the most part, to a rigid and narrow consistency as exemplified by the PC style computer and the centrally managed Learner Management System, such as BlackBoard.

In reflecting upon the prevailing conditions in CALL from the point of view of the researcher, there is clearly a tension between the need for coherence and stability on the one hand - especially for those who see CALL emerging as a legitimate academic pursuit - and the need for nimble-footed adaptation on the other. This tension has been captured in the numerous acronyms that have been suggested as labels to signify the application of the new technologies, hardware and software to language teaching and learning over the last 20 years. 'Why call CALL "CALL"?' discusses this point in depth (Levy \& Hubbard, 2005). Some other acronyms include CALI, CELL, 
TELL, NBLT, WELL and MALL, or ICTs and language learning. ${ }^{3}$ A recent editorial in the ReCALL Journal had in its title, 'CALL, WELL and TELL' as writers struggle to cover the bases (Studzinska-Cavour, 2006). There are many more examples. The acronyms variously point towards the means (computer, technology, network, web, mobile technologies), the role (to assist, to enhance, to provide a base) and the focus (instruction, learning, teaching). All labels have their limitations as CALL over time becomes increasingly multifaceted, especially because of its increasingly diverse material means and modes of delivery (computer, laptop, mobile phone, PDA etc.), and its channels for communication and interaction (email, chat, conferencing, SMS, blogs etc.).

This paper examines these issues in more detail concentrating particularly on the need for stability in CALL research on the one hand and the need for adaptation on the other. It attempts to capture the essential nature of the central tension or dilemma through analysis and discussion, and provides examples of long-term and short-term projects. It closes with some thoughts on planning future CALL research work.

\section{To Build or To Replace}

Essentially, we need to chart a path that moves satisfactorily between stability and innovation. In thinking about this tension initially, it is helpful to look again at the acronyms. The central argument for the use of 'CALL' is the one that favours stability over accuracy of representation. In other words, advocates of 'CALL' as a tag believe it is more important to choose a label that has the potential to stand the test of time rather than a label that may more accurately fit the prevailing technology or mode of learning. Thus, from this standpoint, CALL is preferred to MALL (mobile assisted language learning), for example. Even though MALL might more accurately represent current developments in the use of mobile technologies in language learning, the view that is taken is that replacing a long-standing acronym (CALL) with a new one (MALL) is counterproductive. There are two main reasons for this: the first is that the new technology is not proven and may not take hold; the second is that CALL is diverse and the new acronym may not encompass important CALL activities that already exist and work successfully. Proponents of the alternative viewpoint argue the opposite: that it is more important to more accurately reflect the technology in use or the learning approach in the acronym, thus MALL, WELL or NBLT would be preferred to CALL, and as new technologies were introduced the acronym would be replaced accordingly.

As with the acronyms, in CALL research basically we have the same choice: to build or to replace. Consider the implications, both positive and negative, of each approach. Research that builds is, of course, the traditional approach. We review the literature, the conceptual and theoretical frameworks and we design our study accordingly. Importantly, past experience matters, and it is valued. We assume that the approaches, frameworks and tasks used in earlier projects can be used in new ones. Longitudinal as well as crosssectional studies can be conducted. Sophisticated research designs can be 
attempted. As far as technological innovation is concerned, maybe we are using technology that is relatively stable, such as email or chat, so that we can build on previous research. At other times, we may be using a new technology, but feel able to make the assumption that the research design is not compromised as a result of the technology itself, or the difference can be managed successfully; for example, that we can do a study using SMS on mobile phones in basically the same way as we might conduct a study using email. In either case, we can build on previous research and extend it.

In contrast, consider how we might proceed with a new technological innovation of the kind that has gained wide acceptance in the community at large. As an example, consider MP3 players, which are now readily available at reasonable cost. The author is currently involved in a research project along these lines having noted that over $80 \%$ of students in first year Korean had MP3 players. As there is no previous research to fall back on, initial questions are inevitably exploratory, e.g. What can we use it for in language learning?, What tasks are most appropriate?, How did learners respond?, Do they like it? These are very practical questions. Many are evaluative questions rather than research questions. Often basic connections are made with the language area or skill that is believed to correspond or fit with the technology, so in this case, given the functionality of an MP3 player, there is an obvious link with listening. Identifying, then clarifying this link can help provide a very effective point of departure. Though the technology may be new, research on listening is not. Given that previous research has been conducted on listening, the prospective researcher might begin by reviewing the literature in that area.

With a new, emerging technology the researcher often begins with a pilot study of some kind. Appropriate tasks are prepared that satisfy both pedagogical goals and the functionality or affordances of the technology. Then, typically, the researcher investigates learner attitudes or perceptions, most frequently by using surveys of some kind. It is a little like a consumer survey to see if the customers like it. A particular danger with such research is novelty effects (see Levy \& Stockwell, 2006).

Generally speaking, the researcher's approach and goals tend to differ according to whether the technology is more established or emerging. This difference is neatly captured in Krathwohl's (1993) analysis of the roles and outcomes of research. He suggests a logical, sequential order, namely exploration, description, explanation and validation, as follows:

(1) Exploration: poking around where others haven't, trying something to see what happens, bringing fresh eyes to old situations - discovers new situations and relationships to understand (p. 5).

(2) Description: [Exploration] precedes description, which captures those findings so that we can fit them with explanations and then test or validate those explanations ... It incorporates creativity first in perceiving important aspects of the situation missed by others and second in organizing and presenting that perception so richly and vividly that it comes alive.... (p. 5-6). 
(3) Explanation: ... logically follows from a good description. Explanation may take the form of creatively recognizing a relationship between and among variables... Explanations help us understand situations well enough to predict what will happen in them. In explaining how something works or why a particular phenomenon occurs, we describe the relationships among its parts (p. 6).

(4) Validation: [Having] described the situation, we try to understand and explain what is going on. Finally, we test the explanation to be sure that it works ... it determines whether the predictions based on the explanation will prove accurate (p. 7).

Though, as Krathwohl observes, this order may not in reality be as neatly separated as it appears, it is helpful in conceptualising research involving new technologies.

It is also critical to recognise that new technologies now each have their own developmental histories and chronologies. The label, new technologies, tends to disguise this important fact, especially with the use of the word 'new', which of course is always relative. What was new in 1997 is not necessarily new in 2007. Again we find that labels are crucially important. Arguably technologies such as email are no longer most appropriately classified as 'new'. In fact, new technologies range from the relatively established (e.g. email, chat) to the newly emergent (e.g. podcasting, wikis), a point discussed at length in Levy and Stockwell (2006). If CALL were a larger field, maybe, after all, there would be a case to separate out all the different technologies and give them labels, although there is much overlap between them and the CALL label retains its value as a global, inclusive descriptor. Certainly, however, as far as research is concerned, there is a case for looking at new technologies differentially, either one by one or in various combinations in order to judge their pedagogical value and effectiveness.

\section{Implications for CALL Research}

This section aims to expand on some of these ideas by examining some trends and examples in CALL in more detail. Two specific research studies are used to exemplify key points, one where the goal is primarily to build within a long-standing research agenda, and the other where the goal is to explore the potential value offered by a much more recent new technology. The longerterm perspective and an exemplary project are discussed first.

In Levy and Stockwell (2006), five research areas in CALL were suggested as worthy of consideration in the longer-term, as follows:

(1) Researching language learning through chat at a distance (Tudini, 2003).

(2) Researching intercultural learning through a collaborative email exchange (O'Dowd, 2003).

(3) Researching L2 reading on the Web (Chun, 2001, 2006).

(4) Researching student use of feedback and help (Pujolà, 2002).

(5) Experimental research: choosing the most effective annotation design (Jones, 2003, 2006). 
In the opinion of Levy and Stockwell, the goals and approaches taken in these studies were judged to have sufficient quality, force and potential to drive parallel research agendas; in many cases they are already part of them. An exemplary study was described in each case and was included in parenthesis against each item. These are certainly not the only options, but they have some enduring qualities that make them worthy of serious consideration for longer-term research in CALL.

A good example of a longer-term study embedded in earlier work is provided by Chun on second language (L2) reading in technology-mediated learning contexts (Chun, 2001, 2006). Chun's (2001) study examined how 23 2nd-year German learners accessed information while completing reading tasks using a web-based program called netLearn. The students used a variety of online multimedia resources when completing the tasks including a glossary, an online bilingual dictionary and an audio narration of the text. After reading a text, each student wrote a summary, which was scored according to the number of propositions recalled from the original text. A subset of four students did think-aloud protocols while they were reading online and another group of seven students completed an interview after they had finished the online task; in the interview the questions were designed to investigate the students' metacognitive reading strategies.

This study critically reviewed the substantial body of research on reading, offline and online, in considerable detail. In so doing it moved from a review of the general findings on the reading process to research on reading in hypermedia environments, first more generally and then for L2 reading specifically. It also covered the research on individual differences in learning with hypermedia. The literature review indicated clearly what research had and had not been completed and theoretical and conceptual frameworks that underpin the study were described. This background provided a clear foundation for a series of interrelated, explicitly stated research questions.

In reflecting upon the work of Chun and her colleagues over the last 10 years, one notes the embeddedness of the research in the relevant theory and literature, and the gradual refinement of the research questions over time. This group of studies has moved through all of Krathwohl's stages, from exploration through description and explanation to validation. Chun's ideas have proved sufficiently powerful to be capable of adaptation through technological innovation and change, from standalone software and multimedia, as in the Cyberbuch project (Chun \& Plass, 1997) through to hypermedia learning environments and the Web using netLearn (Chun, 2001, 2006). This capacity to adapt as new technologies are introduced illustrates the strength of CALL research and development that has a strong foundation in learning theory with links to technologyindependent research agendas.

Chun's focus on L2 reading also indicates the value of research that benefits from the rich association between CALL and the language areas and skills. Here language areas are taken to include pronunciation, grammar, vocabulary, discourse and culture (see Levy \& Stockwell, 2006). Recent work on listening (Frommer, 2006; Jones, 2003, 2006) and culture (Furstenberg et al., 2001; 
Levet \& Waryn, 2006; O'Dowd, 2003) provide further convincing examples of the value of the association between the language areas and skills and CALL research (see also Ducate \& Arnold, 2006).

In her recent paper on CALL technologies for L2 reading, Chun (2006) provides suggestions on future directions and a research agenda, plus practical applications. As far as L2 reading research is concerned, Chun clarifies where we stand now, what we know and what we do not. She indicates areas and directions for extending current work such that we can further build and extend our existing knowledge and understanding.

In contrast, the context for the second example discussed here in a little more depth is very different. This study resides chiefly in the exploration phase with regard to Krathwohl's roles for research. It concerns a preliminary study that aimed to assess the potential of mobile phones and SMS messaging for L2 vocabulary learning (Levy \& Kennedy, 2005). Little research had been conducted in this emerging area of mobile learning (see Thornton \& Houser, 2002). Right at the outset attempts were made by the authors to embed the study in what we knew already about vocabulary learning, theory and practice, and the role of technology in this context. The curriculum setting was also a major consideration.

In this case, the authors appealed chiefly to L2 theory and vocabulary learning (Nation, 2001) and a curriculum context to provide a foundation for the study. Note that working with the new technological innovation in an educational context does not preclude the use of existing theoretical, pedagogical and curriculum frameworks, in fact quite the opposite. However, the new technology often does lead to a different set of research goals and questions. The first trial related to a course on Italian Literature and Society and used the technology to provide new vocabulary, presentation and practice, at regular intervals between the scheduled weekly classes. The technology service tested allowed SMS text messages to be prepared in advance and then sent them to class members via conventional e-mail and a PC.

The goals in this exploratory study were rather different in focus, complexity and intent compared to the kind of questions being asked in the earlier example by Chun. In this study, the research questions were more tentative and exploratory, and focused particularly on assessing the value of the functionality of the technology. Levy and Kennedy were especially interested in the following:

- the timing of the messages,

- the optimal interval between messages,

- the value of repeated messages,

- the preferred message content (vocabulary, grammar),

- message comprehensibility,

- use of the L1/L2,

- repetition and recall,

- perceived value, and

- level of imposition. 
Such a list very much represents an exploratory set of research questions best answered, at least initially, through basic data collection instruments such as the questionnaire and the interview.

The last item in the bullet point list is noteworthy. For those interested in the applications of technology in learning, it is always important to keep a close eye on what technologies are being adopted in the wider environment, and to reflect upon the technologies favoured by the population at large with a view to their possible application in education. While there is no reason why the outside environment should dictate or prescribe the technologies used within the educational institution, clearly there are advantages if there is a degree of synchrony between the two. Having a degree of correspondence, when the situation allows it, has two distinct advantages: firstly, less time and attention will need to be allocated to learner training in the institution because students will already be familiar with the technology and will bring that expertise to the classroom; secondly, transfer of knowledge and expertise between the learners' world inside and outside the institution will be much easier. Creating or exploiting a correspondence between formal and informal environments of technology use, between the school and the home, for example, is important when circumstances allow it.

Still, widespread acceptance and use of new communication technologies in the world at large does not necessarily point to effectiveness or value in the educational context. Effective transfer depends to a large degree on the nature of the particular technology, its strengths and limitations, both as a technology and as a pedagogical tool, and the social and cultural conditions that surround its use. There is always a possibility that mobile phone users will see any educationally motivated use of this technology as an unwanted incursion into their own personal, social space and as a result, would strongly reject this kind of usage. On the other hand, within certain parameters and for certain kinds of students such a use might be welcomed. This study aimed to shed more light on this important issue.

\section{Conclusion}

The issues discussed in this paper are central when formulating a CALL research project or agenda because the technology itself, by definition, plays such a pivotal role. Although ideally it is advantageous to try and divorce a specific technology from the context of the research study, often this is not easily accomplished because the qualities and functionality of the technology are inevitably bound up with the focus and direction of the research. While a researcher can stubbornly stay with a technology once it has been superseded, generally this is not advantageous for the obvious reason that in many cases the technology ceases to be available or supported. Examining deeper learning or pedagogical issues can go some way to ameliorating the situation, but often it is not straightforward and the tension at the core of CALL research discussed in this paper at some level remains. 
As far as CALL research is concerned, in fact for all research, I would argue that our goal should always be to build upon what has gone before. This may sound rather obvious, but in CALL, as in many new areas of interdisciplinary research that involve new technologies and applications, this practice can sometimes be difficult to sustain given the continued, regular introduction of new products as the research work continues. In the longer term, adaptation is sometimes possible, as we saw in Chun's work. Other strategies that can assist may involve a series of smaller, but related pilot studies, or a focus on a particular design element or feature that is likely to remain through successive versions or iterations of a technology. In the shorter term, when something new is introduced, we need to critically assess whether it has real potential value for language teaching and learning, or whether it is simply a passing trend with little or no lasting value for teaching and learning. Making clear, objective judgements can sometimes be difficult, especially because of our modern culture's belief in progress where the new innovation is usually preferred to what exists already, whatever the other considerations may be. This preference is often reflected in the decisions made by granting bodies and agencies where the emerging new technology seems to attract funds and where the need to support something older, though still successful, is overlooked.

As far as technological innovation is concerned, there is a strong urge towards replacement. In these circumstances, it is reasonable to conduct principled assessments of new technologies. Such assessment may involve exploratory research or action research. In so doing, though the technology may be new, it is vital to make links with existing CALL practice using prior research studies associated with the language skills or areas, and relevant theories of learning and acquisition. Then, should the new technology gain traction, we will have begun to create a sound footing for further, more focused and intensive research to follow. The kind of research that builds is more likely to contribute to the notion of CALL as a discipline that can contribute more widely to our knowledge and understanding of language learning in a technology-mediated context.

\section{Correspondence}

Any correspondence should be directed to Associate Professor Mike Levy, Head of School, School of Languages and Linguistics, Nathan Campus, Griffith University, 170 Kessels Road, Nathan, Brisbane, Queensland 4111, Australia (michael.levy@griffith.edu.au).

\section{Notes}

1. In this paper, the label 'CALL' for Computer-Assisted Language Learning is used broadly and as a general, inclusive label to include all the acronyms included in Endnote 3. For discussion see Levy and Hubbard (2005).

2. However, we should not forget that the technologies of the kind we are talking about are designed for native speakers of English, and not non-native speakers of the language who do not have a native-speaker-like understanding of the language to fall back on when problems arise. 
3.

\begin{tabular}{|l|l||}
\hline Acronym & \multicolumn{1}{c|}{ Meaning } \\
\hline CALI & Computer-assisted language instruction \\
\hline CALL & Computer-assisted language learning \\
\hline CELL & Computer-enhanced language learning \\
\hline ICTs & Information and communication technologies \\
\hline MALL & Mobile-assisted language learning \\
\hline NBLT & Network-based language teaching \\
\hline TELL & Technology-enhanced language learning \\
\hline WELL & Web-enhanced language learning \\
\hline
\end{tabular}

\section{References}

Chun, D.M. (2001) L2 reading on the Web: Strategies for accessing information in hypermedia. Computer-Assisted Language Learning 14 (5), 367-404.

Chun, D.M. (2006) CALL technologies for L2 reading. In L. Ducate and N. Arnold (eds) Calling on CALL: From Theory and Research to New Directions in Foreign Language Teaching. CALICO Monograph series volume 5 (pp. 69-98). San Marcos, TX: CALICO.

Chun, D.M. and Plass, J.L. (1997) Cyberbuch [Computer software]. New York: St Martin's Press.

Ducate, L. and Arnold, N. (eds) (2006) Calling on CALL: From Theory and Research to New Directions in Foreign Language Teaching. CALICO Monograph series volume 5. San Marcos, TX: CALICO.

Frommer, J. (2006) Wired for sound: Teaching listening via computers and the World Wide Web. In R.P. Donaldson and M.A. Haggstrom (eds) Changing Language Education Through CALL (pp. 67-93). New York: Routledge.

Furstenberg, G., Levet, S., English, K. and Maillet, K. (2001) Giving a virtual voice to the silent language of culture: The Cultura project. Language Learning and Technology 5 (1), 55-102.

Jones, L.C. (2003) Supporting listening comprehension and vocabulary acquisition with multimedia annotations: The students' voice. CALICO Journal 21 (1), 41-65.

Jones, L.C. (2006) Listening comprehension in multimedia environments. In L. Ducate and N. Arnold (eds) Calling on CALL: From Theory and Research to New Directions in Foreign Language Teaching. CALICO Monograph series volume 5 (pp. 99-126). San Marcos, TX: CALICO.

Krathwohl, D.R. (1993) Methods of Educational and Social Science Research: An Integrated Approach. New York: Longman.

Levet, S. and Waryn, S. (2006) Using the web to develop students' in-depth understanding of foreign cultural attitudes and values. In R.P Donaldson and M.A. Haggstrom (eds) Changing Language Education Through CALL (pp. 95-118). New York: Routledge.

Levy, M. and Hubbard, P. (2005) Why call CALL "CALL"? CALL Journal 18 (3), 143149.

Levy, M. and Kennedy, C. (2005) Learning Italian via mobile SMS. In A. KukulskaHulme and J. Traxler (eds) Mobile Technologies for Teaching and Learning. ODL series (pp. 76-83). London: Kogan Page/Taylor and Francis.

Levy, M. and Stockwell, G. (2006) CALL Dimensions: Options and Issues in ComputerAssisted Language Learning. Mahwah, NJ: Lawrence Erlbaum and Associates. 
Nation, I.S.P. (2001) Learning Vocabulary in Another Language. Cambridge: Cambridge University Press.

O'Dowd, R. (2003) Understanding the "other side": Intercultural learning in a Spanish-English e-mail exchange. Language Learning and Technology 7 (2), 118-144.

Pennington, M. (1991) Positive and negative potentials of word processing for ESL writers. System 19 (3), 267-275.

Phillips, M. (1985) Educational technology in the next decade: An ELT perspective. In C. Brumfit, M. Phillips and P. Skehan (eds) Computers and English Language Teaching. ELT Documents 122 (pp. 99-119). Oxford: Pergamon.

Pujolà, J.-T. (2002) CALLing for help: Research in language learning strategies using help facilities in a web-based multimedia program. ReCALL 14 (2), 235- 262.

Studzinska-Cavour, G. (2006) Editorial. CALL, WELL and TELL: Fostering autonomy. ReCALL 18 (1), 1-3.

Thornton, P. and Houser, C. (2002) M-learning: Learning in transit. In P. Lewis (ed.) The Changing Face of CALL: A Japanese perspective (pp. 229-244). The Netherlands: Swets and Zeitlinger.

Tudini, V. (2003) Using native speakers in chat. Language Learning and Technology 7 (3), $141-159$. 